\title{
The ongoing outburst of the new symbiotic star IPHASJ190832.31+051226.6 ${ }^{\star}$
}

\author{
R. L. M. Corradi ${ }^{1,2}$, U. Munari ${ }^{3}$, R. Greimel ${ }^{4}$, M. M. Rubio-Díez 5 , M. Santander-García ${ }^{5,1,2}$, P. Rodríguez-Giil ${ }^{5,1,2}$, \\ J. E. Drew ${ }^{6}$, P. Leisy ${ }^{5}$, T. Liimets ${ }^{7,8}$, and S. E. Sale ${ }^{9}$ \\ 1 Instituto de Astrofísica de Canarias, 38200 La Laguna, Tenerife, Spain \\ e-mail: rcorradi@iac.es \\ 2 Departamento de Astrofísica, Universidad de La Laguna, 38205 La Laguna, Tenerife, Spain \\ 3 INAF, Osservatorio Astronomico di Padova, via dell'Osservatorio 8, 36012 Asiago (VI), Italy \\ 4 Institut für Physik, Karl-Franzen Universität Graz, Universitätsplatz 5, 8010 Graz, Austria \\ 5 Isaac Newton Group of Telescopes, Apart. de Correos 321, 38700 Santa Cruz de la Palma, Spain \\ ${ }^{6}$ Centre for Astrophysics Research, STRI, University of Hertfordshire, College Lane, Hatfield, AL10 9AB, UK \\ 7 Nordic Optical Telescope, Apart. de Correos 474, 38700 Santa Cruz de la Palma, Spain \\ 8 Tartu Observatoorium, Tõravere 61602, Estonia \\ 9 Astrophysics Group, Imperial College of Science, Blackett Laboratory, Prince Consort Road, London SW7 2AZ, UK
}

Received 12 November 2009 / Accepted 22 December 2009

\section{ABSTRACT}

\begin{abstract}
Aims. Eleven new symbiotic stars have recently been discovered with IPHAS, the INT H $\alpha$ survey of the Northern Galactic plane. The star IPHAS J190832.31+051226.6 was proposed as an additional candidate on the basis of the existing spectrum. Here, we investigate the nature of this source by means of additional observations.

Methods. Photometric data, optical spectra obtained in 2006 and 2009, a higher resolution spectrum resolving the $\mathrm{H} \alpha$ profile, and near-IR spectra of IPHAS J190832.31+051226.6 are all presented.

Results. The source brightened in the $r$ band by 2.3 mag from 2004 to 2009. From 2006 to 2009, the spectrum has evolved from one with the obvious continuum of an M giant star plus HI and HeI lines in emission to a lower excitation nebular spectrum with HI, CaII, and FeII emission and a bluer continuum in which the absorption bands of the red giant are only visible at wavelengths longer than $7500 \AA$. The $\mathrm{H} \alpha$ line is broad with a deep central absorption and extended wings.

Conclusions. The averaged rate of the brightness increase, the rise of a blue continuum overwhelming the absorption bands of the $\mathrm{M}$ giant, and the corresponding decline of the ionization condition of the emission-line spectrum, are all consistent with the hypothesis that IPHAS J190832.31+051226.6 is a new symbiotic star picked up during the onset of a symbiotic nova outburst that is still in progress at the time of writing.
\end{abstract}

Key words. binaries: symbiotic

\section{Introduction}

IPHAS, the $\mathrm{H} \alpha$ survey of the Northern Galactic Plane IPHAS (Drew et al. 2005), is providing a wealth of new information about the population of $\mathrm{H} \alpha$ emitting stars in the Milky Way. Among them, Corradi et al. $(2008,2010)$ present the discovery of eleven symbiotic stars, doubling the number of objects of this class known in this area of the sky. Corradi et al. (2010) indicate two additional candidates: one of them - named IPHAS J190832.31+051226.6 according to its J2000 coordinates - was noticed to have brightened significantly from 2004 to 2008. This raised our interest in the source, so we obtained new photometric and spectroscopic data that are presented here.

\section{Observational data}

Photometry of IPHAS J190832.31+051226.6 from 2004 to 2009 in the Sloan $r$ and $i$ bands has been extracted from multi-epoch

* Based on observations obtained at; the $2.6 \mathrm{~m}$ Nordic Optical Telescope operated by NOTSA, and the $2.5 \mathrm{~m}$ INT and $4.2 \mathrm{~m}$ WHT telescopes of the Isaac Newton Group of Telescopes in the Spanish Observatorio del Roque de Los Muchachos of the Instituto de Astrofísica de Canarias. images obtained with the Wide Field Camera at the $2.5 \mathrm{~m}$ Isaac Newton Telescope (INT) on La Palma, Spain. Data were obtained during the IPHAS survey, with an additional observation secured during a recent run of the (IPHAS-related) UVEX survey (Groot et al. 2009), and another one extracted from the INT archive. As the global photometric calibration of IPHAS is not yet available, the magnitudes of IPHAS J190832.31+051226.6 at different epochs have been determined using a large number of field stars. As a result, the magnitude variations of IPHAS J190832.31+051226.6 should be considered as accurate to within \pm 0.05 mag (including the photometric error in the measurement of the target), but the whole set of magnitudes might have a small offset (less than $0.1 \mathrm{mag}$ ) from the correct zero point. In addition, the POSSII red ( $F$-band) image obtained in 1987 was inspected, and the Sloan $r$ mag of the source was estimated by comparison with nearby stars, applying the transformations between the different photometric systems as described in Sesar et al. (2006) and in the Cambridge Astronomical Survey Unit (http://www.ast.cam.ac.uk/ wfcsur). The error associated with this early photometric point is estimated to be $0.2 \mathrm{mag}$.

A 30 min spectrum of IPHAS J190832.31+051226.6 was obtained using the IDS spectrograph at the INT on 9 September 


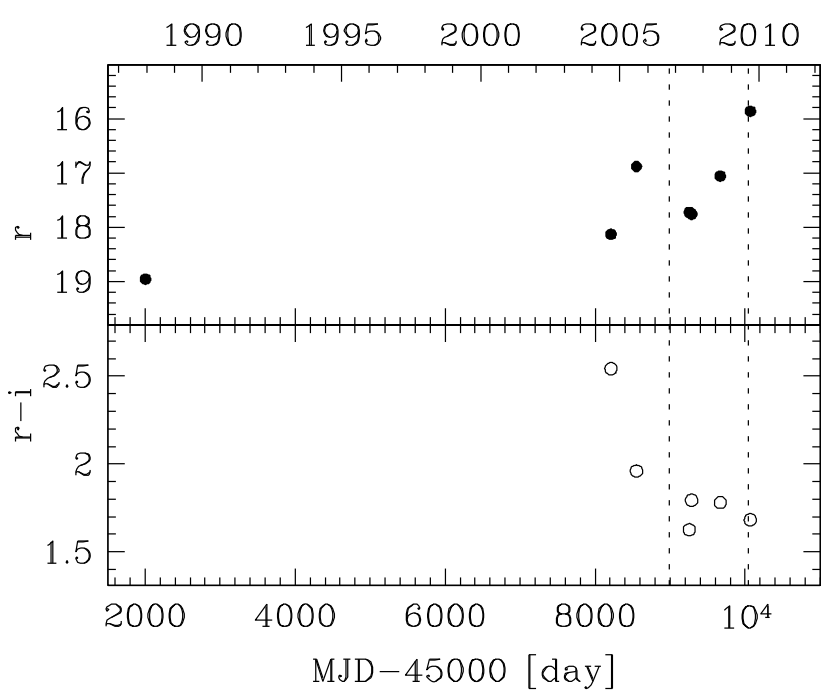

Fig. 1. Photometric data of IPHAS J190832.31+051226.6. Vertical dashed lines show the dates when the spectra in Fig. 2 were obtained. The errors on the data points for the last 5 years in the upper panel are smaller than the plotted points. In the lower panel they are $<0.1$.

2006 as part of the International Time Programme awarded to the IPHAS collaboration. The R300V grating was used with a slit width of 1.1 arcsec. This results in a spectral resolution of $5.0 \AA$ with a dispersion of $1.9 \AA$ pix $^{-1}$ over a useful spectral range from 4700 to $8000 \AA$. A second optical spectrum was obtained during the morning of 29 July 2009 at the $4.2 \mathrm{~m}$ WHT telescope on La Palma with the ISIS spectrograph. In the blue arm, grating R600B was used in combination with a 1". 5 arcsec wide slit, providing a dispersion of $1.73 \AA \mathrm{pix}^{-1}$, a resolution of $5 \AA$, and a spectral coverage from 3700 to $5350 \AA$. In the red arm, grating $\mathrm{R} 158 \mathrm{R}$ was used, giving a dispersion of $3.64 \AA \mathrm{pix}^{-1}$, a resolution of $9.5 \AA$, and spectral coverage from 5550 up to $9200 \AA$. The total exposure time was $35 \mathrm{~min}$ in each arm. The signal-tonoise ratio $(\mathrm{S} / \mathrm{N})$ in the continuum around $\mathrm{H} \alpha$ for the 2006 and 2009 optical spectra is 10 and 20, respectively, increasing from blue to red owing to the cool continuum of the source.

With the same telescope, we secured a near-IR spectrum on 28 August 2009 using the LIRIS spectrograph covering the range from 0.9 to $2.4 \mu \mathrm{m}$. A slit width of 0.75 and the low-resolution $z J$ and $H K$ grisms provided a resolution of $12 \AA$ and $20 \AA$, respectively. Exposure time was $400 \mathrm{~s}$ in the $z J$ grism and $200 \mathrm{~s}$ in the $H K$ one. A B9 V star was observed at the same airmass as the target, and used for telluric correction and flux calibration. The $\mathrm{S} / \mathrm{N}$ is between 20 and 40 .

Finally, a 20 min higher resolution spectrum centred on the $\mathrm{H} \alpha$ line was obtained at the $2.6 \mathrm{~m}$ Nordic Optical Telescope on La Palma on 17 August 2009. The ALFOSC spectrograph was used in combination with grism \#17 and a 0.5 wide slit. This setup covers the region from 6350 to $6850 \AA$ with $0.65 \AA$ resolution. The $\mathrm{S} / \mathrm{N}$ in the continuum is around 10 .

\section{Analysis}

Figure 1 shows the evolution of the brightness of IPHAS J190832.31+051226.6. The source was fainter in $1987(r \sim 19.0 \mathrm{mag})$ than in the first IPHAS observation of 2004 ( $r=18.13 \mathrm{mag}$ ), after which it brightened up to the present $r=15.86$ mag and became almost one magnitude bluer in the $r-i$ colour. There are not many data points, but it is clear that the magnitude rise might have started before 2004, that it is not monotonic, and that it is slow at an averaged rate of

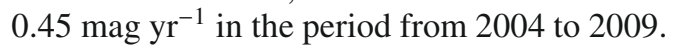

The 2006 spectrum of the source (Fig. 2, upper panel) consisted of a red continuum with relatively deep $\mathrm{TiO}$ bands at $6160,6569,7053,7589$ and $7666 \AA$ typical of a mid-M giant. On the top of the cool star continuum, a strong and relatively broad $\mathrm{H} \alpha$ emission line was observed. Its flux was $3.8 \mathrm{e}-14 \mathrm{erg} \mathrm{cms}^{-2} \mathrm{~s}^{-1}$, and the full width at half maximum, corrected for the instrumental profile, was $250 \mathrm{~km} \mathrm{~s}^{-1}$ with additional extended wings. The spectrum also presented HeI emission lines at 5876, 6678, 7065, and $7281 \AA$, a weak $\mathrm{H} \beta$ roughly 70 times fainter than $\mathrm{H} \alpha$, and marginal evidence of the [O III]5007 forbidden line. The 2006 INT spectrum of IPHAS J190832.31+051226.6 does not extend enough into the blue to cover the HeII $4686 \AA$ line. The lack of a clear detection of high excitation nebular lines led Corradi et al. (2010) to flag the source as a "possible" symbiotic star.

The more recent optical spectrum taken in 2009 (Fig. 2, lower panel) is characterized by a blue and bright continuum overwhelming the $\mathrm{TiO}$ bands of the $\mathrm{M}$ giant. The latter are only visible at $\lambda \geq 7500 \AA$. Strong diffuse interstellar bands (DIBs) and NaID interstellar lines are now visible in absorption on the otherwise featureless continuum. Concerning emission lines, the HeI lines have disappeared, and several low ionization FeII lines appeared together with a strong near-IR CaII triplet. The $\mathrm{H} \alpha$ flux is basically unaltered from 2006, but the line is broader, and $\mathrm{H} \beta$ did not change either, within the large errors associated with the measurement of this weak line.

The 2009 near-IR spectrum (Fig. 3) provides further information for classification of the cool giant. At these wavelengths, the spectrum seems dominated by the cool star, with relatively faint and broad HI Paschen $\beta$ and $\gamma$ emission lines. The most prominent features of the red giant are the TiO bands with band-head at $1.1 \mu \mathrm{m}$, and the strong CO series whose depth unambiguously proves the giant luminosity class of the star - beginning at 1.56 and $2.29 \mu \mathrm{m}$. Judging from the strength of the observed $\mathrm{TiO}$ absorption band, and the absence of both the $\mathrm{TiO}$ band at $0.97 \mu \mathrm{m}$ and the $\mathrm{VO}$ band at $1.046 \mu \mathrm{m}$, the type of the cool star should lie between M3 and M5 (Wright et al. 2009). Also, from the equivalent widths of the CO band at $2.29 \mu \mathrm{m}$, the Na I doublet at $2.208 \mu \mathrm{m}$, and the $\mathrm{Ca}$ I triplet at $2.265 \mu \mathrm{m}$, we estimate a solar-like metallicity $[\mathrm{Fe} / \mathrm{H}]=0.03 \pm 0.23$ (Ramirez et al. 2000), and a stellar $T_{\text {eff }}$ of $3600 \pm 140 \mathrm{~K}$ (Ramirez et al. 1997). These correspond to an M4 or M5 type (Mármol-Queraltó et al. 2008). The fit of the 2006 optical spectrum of Fig. 2 following the procedure described in Corradi et al. (2010) constrains the spectral type of the giant to lie between M4.0 III (reddened by $E_{B-V}=1.70$ ) and M6.0 III $\left(E_{B-V}=1.55\right)$.

Combining all the different indicators, the red giant of IPHAS J190832.31+051226.6 is constrained to between early M4 III and late M5 IIII. With the calibration into absolute magnitudes of $\mathrm{M}$ giants as discussed in Corradi et al. (2010), a distance for IPHAS J190832.31+051226.6 between 3.3 and $5.4 \mathrm{kpc}$ is obtained. The range of possible distances and reddening values is plotted in Fig. 4 and is compared with the extinctiondistance relationship along the line of sight to the source provided by IPHAS (see Sale et al. 2008; and Corradi et al. 2010). In the figure, empty circles indicate the curve derived using hundreds of field stars in a $10^{\prime} \times 10^{\prime}$ box around the line of sight toward IPHAS J190832.31+051226.6. Beyond a distance 


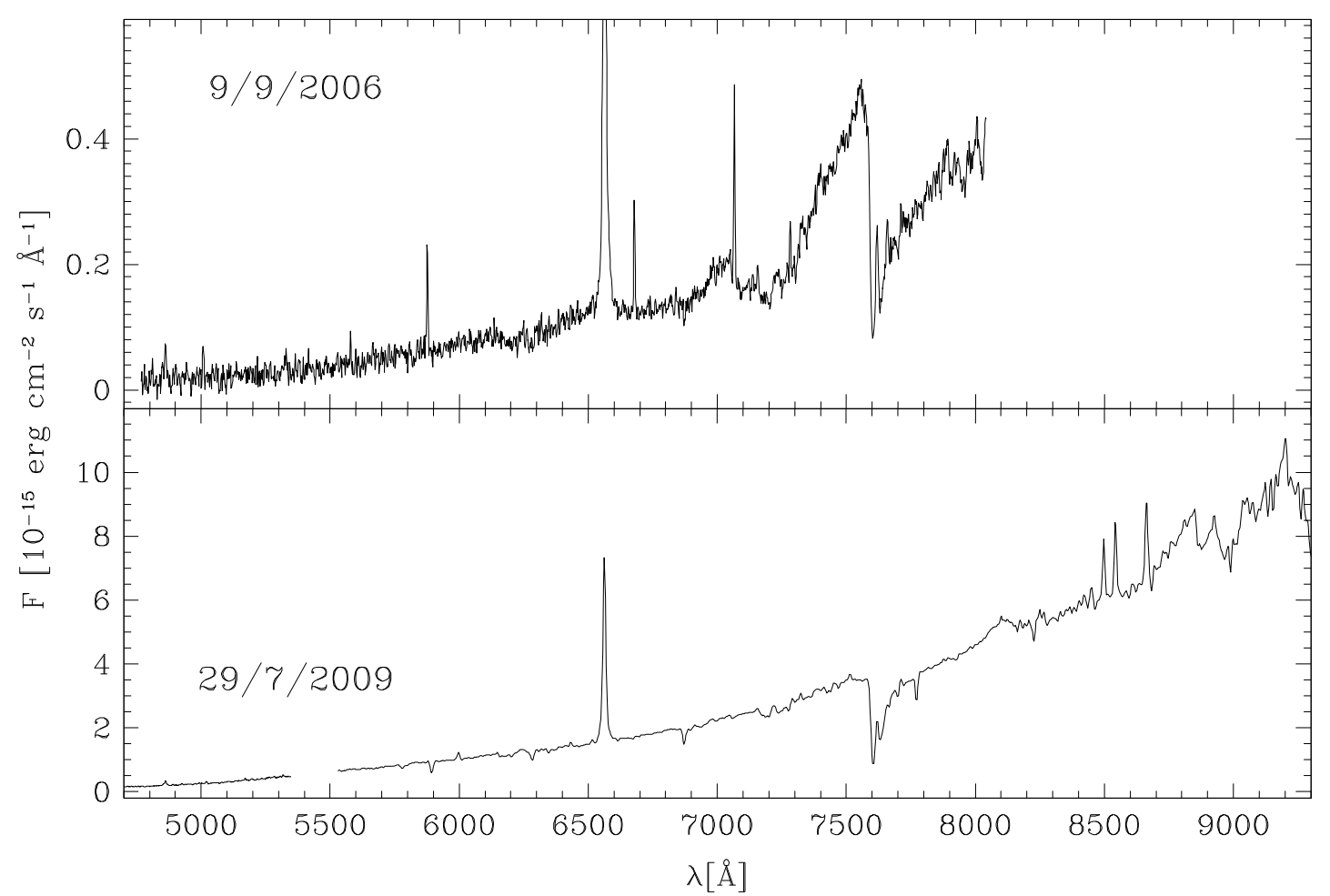

Fig. 2. 2006 and 2009 optical spectra of IPHAS J190832.31+051226.6, with different $y$-scales in the two panels.

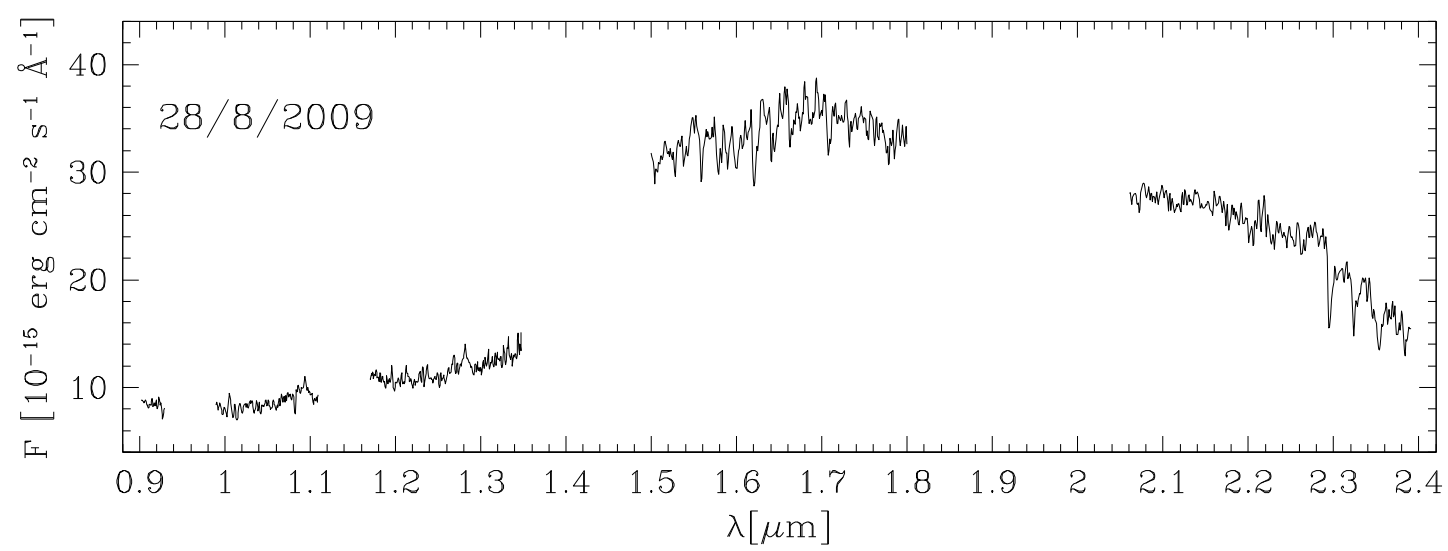

Fig. 3. 2009 near-IR spectrum of IPHAS J190832.31+051226.6. The spectral regions affected by strong atmospheric absorption are not displayed.

of $3.3 \mathrm{kpc}$, there are not enough stars to build the curve, but 2MASS data (Marshall et al. 2006) suggest that reddening continues to rise linearly until $4 \mathrm{kpc}$, and then more slowly till at least $12 \mathrm{kpc}$. If no significant circumstellar reddening is present, these extinction constraints are consistent with a distance to IPHAS J190832.31+051226.6 of around $4.5 \mathrm{kpc}$, combined with a spectral type of M5 III.

17 August 2009. Its shape is typical of symbiotic stars (cf. van Winckel, Duerbeck and Schwarz 1993, where the most similar profiles are those of BI Cru and AS 296), and the deep and sharp central reversal is often associated with outbursting systems. There are broad wings extending to more than $\pm 500 \mathrm{~km} \mathrm{~s}^{-1}$.

IPHAS J190832.31+051226.6 was detected in quiescence by the 2MASS infrared survey at magnitudes $J=10.685$ $( \pm 0.016), H=9.261( \pm 0.017), K=8.531( \pm 0.006)$ on JD 2451401.6 (11 Aug. 1999). In the 2MASS colour-colour diagram, IPHAS J190832.31+051226.6 lies slightly to the right of the sequence of reddened RGB stars, showing a modest
IR-excess, in a region mainly occupied by S-type symbiotic stars but with some D-types also present (see Corradi et al. 2008, 2010).

\section{Conclusions}

From its photometric and spectroscopic properties, we conclude that IPHAS J190832.31+051226.6 is a new symbiotic star and that it is observed during the luminosity rise of a symbiotic nova outburst. This conclusion is based on the main following arguments.

Its spectrum is a combination of the radiation from a mid$\mathrm{M}$ giant star and a hotter component producing the observed emission-line spectrum (including HI and HeI in 2006). Only the lack of any clear detection of high excitation lines (either because of the low $\mathrm{S} / \mathrm{N}$, high reddening, or high electron density) prevents an immediate classification as a symbiotic star. The circumstellar gas is characterized by a very high electron density, 


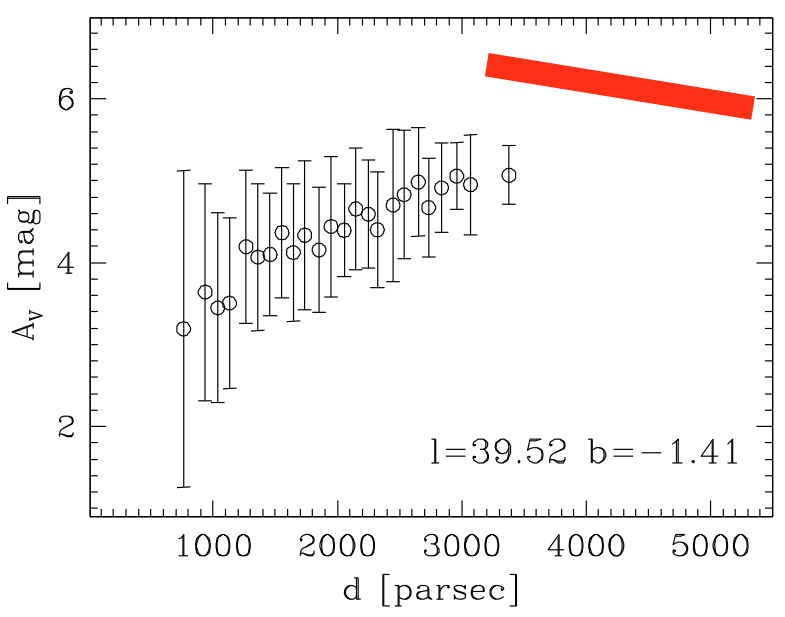

Fig. 4. Interstellar extinction vs. distance for the sightline toward IPHAS J190832.31+051226.6. The broad (red) line indicates the range of possible distance and extinction values determined from modelling the 2006 spectrum: its left end corresponds to an M4.0 III type and $E(B-V)=1.69$, and its right end to M6.0 III and $E(B-V)=1.55$.

as indicated by the ratio of the HeI lines at 5876 and $6678 \AA$. The ratio is close to 1.0 and corresponds to electron densities $N_{\mathrm{e}} \geq 10^{10} \mathrm{~cm}^{-3}$ (Schmid 1989). Also, the nebular continuum dominates the spectrum of the $\mathrm{M}$ giant blue-ward of $\mathrm{H} \alpha$ even in 2006, when the system was at or near quiescence. This requires a high photo-ionization luminosity of the companion to the giant, of at least several $10^{2} L_{\odot}$ (Murset et al. 1991). All the above is typical of symbiotic stars: Ap 3-1 or V2110 Oph are examples of genuine symbiotics with an optical spectrum closely matching that of IPHAS J190832.31+051226.6 in 2006 (cf. Munari \& Zwitter 2002).

In symbiotic stars, the amplitude of the outburst decreases with wavelength, because of the increasing contribution of the cool giant that is not affected by the outburst (Siviero et al. 2009). In typical outbursts, the amplitude in the B band is between 2 and $4 \mathrm{mag}$, while it does not exceed one magnitude in the Rc band. The amplitude of 3 mag in the $r$ band displayed by IPHAS J190832.31+051226.6 is instead significantly larger. Also, in classical symbiotic star outbursts, the luminosity rise toward maximum is fast, usually lasting a few months (e.g. Munari et al. 2009a), while IPHAS J190832.31+051226.6 could still be brightening five years after the start of the IPHAS photometric monitoring. A large amplitude in the red and a very slow rise to maximum are more appropriate to "symbiotic novae", also called "slow novae". These are symbiotic stars that have experienced only one outburst over historic times, lasting a few decades or more. One example is V4368 Sgr (=Wakuda's peculiar variable; Munari et al. 2009b) that took no less than four years to reach the optical maximum and where the amplitude in the red largely exceeded the one displayed (so far) by IPHAS J190832.31+051226.6. Other examples are V1329 Cyg, in which the system took 7 years to get to its maximum visual luminosity (Munari et al. 1988), and RX Pup (10 years, Mikolajewska et al. 1999). IPHAS J190832.31+051226.6 seems to have a luminosity development similar to these symbiotic novae, but the sparse photometric data available so far do not allow us to precisely determine the structure of the outburst and to disentangle it from secular changes. A higher cadence monitoring in the coming years is needed.

The spectroscopic behaviour during outburst of IPHAS J190832.31+051226.6 is also typical of symbiotic

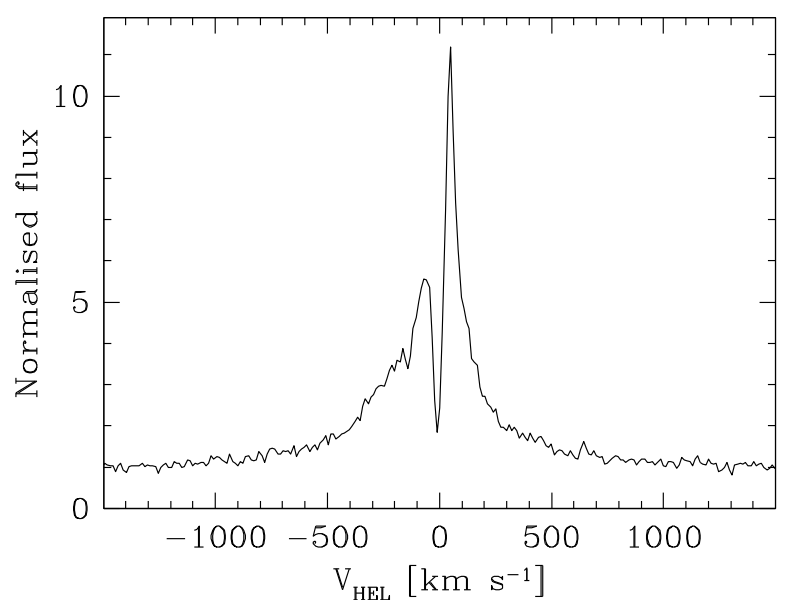

Fig. 5. H $\alpha$ profile of IPHAS J190832.31+051226.6 on 17 August 2009.

stars: (i) disappearance of the higher ionization/excitation emission lines; (ii) appearance of a blue continuum from the outbursting component, which overwhelms both the nebular continuum and the molecular absorption spectrum of the cool giant, up to the red region of the spectrum.

Finally, the $\mathrm{H} \alpha$ profile displayed by IPHAS J190832.31+051226.6 is a close match to the profile of CI Cyg during its current outburst (Siviero et al. 2009). Indeed, both have a broad emission component from the circumstellar gas with superimposed a sharp and blue-shifted absorption (tracing the absorbing wind of the $\mathrm{M}$ giant), extended wings, and an asymmetric higher velocity weak component.

Acknowledgements. R.L.M.C. and M.S.G. acknowledge funding from the Spanish AYA2007-66804 grant.

\section{References}

Corradi, R. L. M., Rodriguez-Flores, E. R., Mampaso, A., et al. 2008, A\&A, 480, 409

Corradi, R. L. M., Valentini, M., Munari, U., et al. 2010, A\&A, 509, A41

Drew, J., Greimel, R., Irwin, M. J., et al. 2005, MNRAS, 362, 753

Fitzpatrick, E. L. 1999, PASP, 111, 63

Groot, P. J., Verbeek, K., Greimel, R., et al. 2009, MNRAS, 399, 323

Kenyon, S. J. 1986, The Symbiotic Stars (Cambridge University Press)

Kirkpatrick, J. D., Henry, T. J., \& McCarthy, D. W. 1991, ApJS, 77, 417

Mármol-Queraltó, E., Cardiel, N., Cenarro, A. J., et al. 2008, A\&A, 489, 885

Marshall, D. J., Robin, A. C., Reylé, C., Schultheis, M., \& Picaud, S. 2006, A\&A, 453, 635

Mikolajewska, J., Brandi, E., Hack, W., et al. 1999, MNRAS, 305, 190

Munari, U., \& Zwitter, T. 2002, A\&A, 383, 188

Munari, U., Margoni, R., \& Mammano, A. 1988, A\&A, 202, 83

Munari, U., Siviero, A., Ochner, P., Wallerstein, G., et al. 2009a, PASP, 121, 1070

Munari, U., Ochner, P., Siviero, A., et al. 2009b, BaltA, 18, 75

Mürset, U., Nussbaumer, H., Schmid, H., \& Vogel, M. 1991, A\&A, 248, 458

Ramírez, S. V., Depoy, D. L., Frogel, J. A., Sellgren, K., \& Blum, R. D. 1997, AJ 113, 1411

Ramírez, S. V., Stephens, A. W., Frogel, J. A., \& DePoy, D. L. 2000, AJ, 120, 833

Sale, S. E., Drew, J. E., Unruh, Y. C., et al. 2008, MNRAS, 392, 497

Schmid, H. M. 1989, in Physics of Classical Novae, IAU Coll., 122, Springer, 303

Sesar, B., Svilkovic, D., Ivezic, Z., et al. 2006, AJ, 131, 2801

Siviero, A., Munari, U., Dallaporta, S., et al. 2009, MNRAS, 399, 2139

van Winckel, H., Duerbeck, H. W., \& Schwarz, H. E. 1993, A\&AS, 102, 401

Wright, N. J., Barlow, M. J., Greimel, R., et al. 2009, MNRAS, 400, 141 